\title{
Application of new $400 \mathrm{MHz}$ high temperature superconducting (HTS) power-driven magnet NMR technology for online
} reaction monitoring: Proof of concept with a ring closing

\section{metathesis $(\mathrm{RCM})$ reaction}

\author{
Maria Victoria Silva Elipe, ${ }^{1 *}$ Alan Cherney, ${ }^{2}$ Robert Krull, ${ }^{3}$ Neil Donovan, ${ }^{3}$ Jason Tedrow, ${ }^{2}$ \\ Donald Pooke, ${ }^{4}$ and Kimberly L. Colson ${ }^{5}$
}

${ }^{1}$ Department of Attribute Sciences, Amgen Inc., One Amgen Center Drive, Thousand Oaks, CA USA 91320-1799

${ }^{2}$ Department of Drug Substance Technologies, Amgen Inc., 360 Binney Street, Cambridge, MA USA 02142

${ }^{3}$ Bruker BioSpin, 15 Fortune Drive, Billerica, MA USA 01821

${ }^{4}$ HTS-110, 1B Quadrant Drive, Lower Hutt, New Zealand

${ }^{5}$ Consulting: NMR Spectroscopy, 12 MacQuarrie Lane, Westford, MA USA 01886

*To whom correspondence should be addressed

Maria Victoria Silva Elipe (ORCID ID: https://orcid.org/0000-0002-8429-9534)

Tel: 805-447-9807

E-mail: melipe@amgen.com.

Department of Attribute Sciences, Amgen Inc. 


\section{Supporting Information}

Table of Contents

Table S1. Table of data for kinetic rate calculations for Table 1 and Figure 8.

Figure S1. First 1D WET ${ }^{1} \mathrm{H}$ NMR spectrum of the reaction for Table 1 calculations.... S5

Table S2. Table of data for kinetic rate calculations for Table 1 based on integral ratios from the

first spectrum

S6

Figure S2. Stack 1D WET ${ }^{1} \mathrm{H}$ NMR spectra and kinetic graph for Table 1 calculations based on integral ratios from the first

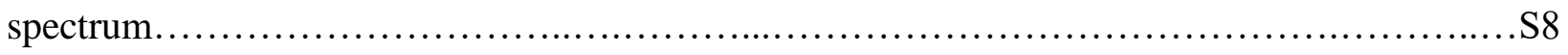

Figure S3. Last 1D WET ${ }^{1} \mathrm{H}$ NMR spectrum of the reaction for Table 1 calculations

Table S3. Table of data for kinetic rate calculations for Table 1 based on integral ratios from the last spectrum. S10

Figure S4. Stack 1D WET ${ }^{1} \mathrm{H}$ NMR spectra and kinetic graph for Table 1 calculations based on integral ratios from the last spectrum.

Figure S5. Results from Bruker Dynamic Center software calculations for Table 1. $\mathrm{S} 13$ 
Table S1. Table of data for kinetic rate calculations for Table 1 and for Figure 8.

Table of data for Figure 8 in the manuscript for kinetic rate calculations using Mnova software. The results of the rate constants are in Table 1 in the manuscript. In this case the concentration is not adjusted considering the concentration of the substrate in the first spectrum as intact.

\begin{tabular}{|c|c|c|c|c|c|}
\hline $\begin{array}{l}\# \\
\text { (spectra) }\end{array}$ & $\begin{array}{l}X(\mathrm{I}) \text { (time in } \\
\mathrm{sec})\end{array}$ & $\begin{array}{l}\mathrm{Y}(\mathrm{X}) \text { (concentration of } \\
\text { substrate in } \mathrm{mM} \text { ) }\end{array}$ & $\begin{array}{l}Y^{\prime}(X) \text { (fitted } \\
\text { data) }\end{array}$ & $\begin{array}{l}\mathrm{Y} 1(\mathrm{X}) \text { (concentration of } \\
\text { product in } \mathrm{mM} \text { ) }\end{array}$ & $\begin{array}{l}\text { Y1'(X) (fitted } \\
\text { data) }\end{array}$ \\
\hline Model & ARR_DATA(I) & Concentration $(2.703,2.540)$ & $\begin{array}{l}B+F^{*} \exp (- \\
\left.x^{*} G\right) \\
B=20.6176 \\
F=77.0075 \\
G= \\
0.000271488\end{array}$ & Concentration $(3.073,2.900)$ & $\begin{array}{l}B+F^{*} \exp (- \\
\left.x^{*} G\right) \\
B=86.9857 \\
F=-80.4395 \\
G= \\
0.000274216\end{array}$ \\
\hline 1 & 0.000 & 94.500 & 97.625 & 5.196 & 6.546 \\
\hline 2 & 146.000 & 96.712 & 94.632 & 8.827 & 9.703 \\
\hline 3 & 289.000 & 93.050 & 91.814 & 12.078 & 12.675 \\
\hline 4 & 496.000 & 89.126 & 87.923 & 16.661 & 16.776 \\
\hline 5 & 640.000 & 85.910 & 85.343 & 19.168 & 19.494 \\
\hline 6 & 782.000 & 83.709 & 82.895 & 22.036 & 22.071 \\
\hline 7 & 924.000 & 80.479 & 80.540 & 24.582 & 24.551 \\
\hline 8 & 1128.000 & 76.949 & 77.311 & 28.093 & 27.947 \\
\hline 9 & 1271.000 & 74.649 & 75.153 & 30.413 & 30.218 \\
\hline 10 & 1413.000 & 72.972 & 73.090 & 32.395 & 32.386 \\
\hline 11 & 1607.000 & 69.683 & 70.398 & 35.329 & 35.214 \\
\hline 12 & 1750.000 & 67.821 & 68.502 & 37.334 & 37.205 \\
\hline 13 & 1893.000 & 66.342 & 66.679 & 39.590 & 39.119 \\
\hline 14 & 2087.000 & 63.682 & 64.316 & 42.122 & 41.599 \\
\hline 15 & 2229.000 & 61.760 & 62.663 & 44.010 & 43.332 \\
\hline 16 & 2372.000 & 61.168 & 61.062 & 45.960 & 45.011 \\
\hline 17 & 2514.000 & 59.873 & 59.533 & 47.522 & 46.614 \\
\hline 18 & 2715.000 & 57.576 & 57.466 & 49.724 & 48.779 \\
\hline 19 & 2857.000 & 56.332 & 56.073 & 51.317 & 50.238 \\
\hline 20 & 2999.000 & 54.486 & 54.732 & 52.446 & 51.642 \\
\hline 21 & 3189.000 & 52.972 & 53.017 & 54.499 & 53.436 \\
\hline 22 & 3331.000 & 51.710 & 51.791 & 55.680 & 54.717 \\
\hline 23 & 3473.000 & 50.473 & 50.612 & 56.600 & 55.950 \\
\hline 24 & 3662.000 & 49.238 & 49.112 & 58.059 & 57.517 \\
\hline 25 & 3803.000 & 47.996 & 48.042 & 59.218 & 58.635 \\
\hline 26 & 3946.000 & 47.069 & 46.998 & 60.074 & 59.725 \\
\hline 27 & 4087.000 & 46.016 & 46.007 & 61.016 & 60.759 \\
\hline 28 & 4293.000 & 44.556 & 44.626 & 62.433 & 62.199 \\
\hline 29 & 4435.000 & 43.574 & 43.718 & 63.385 & 63.146 \\
\hline 30 & 4577.000 & 43.111 & 42.845 & 64.153 & 64.056 \\
\hline 31 & 4780.000 & 41.316 & 41.653 & 65.119 & 65.298 \\
\hline 32 & 4923.000 & 40.756 & 40.852 & 65.774 & 66.132 \\
\hline 33 & 5065.000 & 40.034 & 40.086 & 66.506 & 66.928 \\
\hline 34 & 5251.000 & 39.182 & 39.128 & 67.626 & 67.926 \\
\hline 35 & 5394.000 & 38.343 & 38.423 & 68.060 & 68.659 \\
\hline 36 & 5537.000 & 37.860 & 37.745 & 68.887 & 69.363 \\
\hline 37 & 5679.000 & 37.048 & 37.097 & 69.368 & 70.036 \\
\hline 38 & 5885.000 & 35.971 & 36.201 & 70.664 & 70.967 \\
\hline 39 & 6027.000 & 36.138 & 35.612 & 71.269 & 71.579 \\
\hline 40 & 6169.000 & 35.270 & 35.044 & 71.777 & 72.167 \\
\hline 41 & 6361.000 & 34.395 & 34.312 & 72.428 & 72.927 \\
\hline 42 & 6503.000 & 33.801 & 33.794 & 72.937 & 73.464 \\
\hline 43 & 6645.000 & 33.340 & 33.296 & 73.292 & 73.981 \\
\hline 44 & 6836.000 & 32.660 & 32.655 & 74.066 & 74.644 \\
\hline 45 & 6978.000 & 32.295 & 32.200 & 74.509 & 75.116 \\
\hline
\end{tabular}




\begin{tabular}{|c|c|c|c|c|c|}
\hline 46 & 7120.000 & 31.900 & 31.762 & 75.101 & 75.569 \\
\hline 47 & 7262.000 & 31.144 & 31.340 & 75.328 & 76.005 \\
\hline 48 & 7461.000 & 30.976 & 30.776 & 76.053 & 76.588 \\
\hline 49 & 7604.000 & 30.531 & 30.389 & 76.334 & 76.988 \\
\hline 50 & 7747.000 & 30.082 & 30.017 & 76.570 & 77.372 \\
\hline 51 & 7933.000 & 29.552 & 29.555 & 76.949 & 77.850 \\
\hline 52 & 8075.000 & 29.669 & 29.217 & 77.550 & 78.199 \\
\hline 53 & 8217.000 & 28.858 & 28.891 & 77.589 & 78.535 \\
\hline 54 & 8412.000 & 28.153 & 28.465 & 78.397 & 78.975 \\
\hline 55 & 8554.000 & 28.247 & 28.168 & 78.452 & 79.281 \\
\hline 56 & 8697.000 & 27.983 & 27.880 & 78.722 & 79.577 \\
\hline 57 & 8839.000 & 27.567 & 27.606 & 79.074 & 79.860 \\
\hline 58 & 9037.000 & 27.496 & 27.240 & 79.732 & 80.237 \\
\hline 59 & 9179.000 & 27.037 & 26.990 & 79.988 & 80.494 \\
\hline 60 & 9322.000 & 26.865 & 26.747 & 80.206 & 80.744 \\
\hline 61 & 9518.000 & 26.631 & 26.429 & 80.462 & 81.071 \\
\hline 62 & 9660.000 & 26.217 & 26.210 & 80.468 & 81.296 \\
\hline 63 & 9803.000 & 26.136 & 25.997 & 80.995 & 81.515 \\
\hline 64 & 10003.000 & 25.979 & 25.712 & 81.704 & 81.807 \\
\hline 65 & 10145.000 & 25.952 & 25.520 & 81.684 & 82.005 \\
\hline 66 & 10288.000 & 25.555 & 25.333 & 82.036 & 82.197 \\
\hline 67 & 10430.000 & 25.744 & 25.155 & 82.203 & 82.379 \\
\hline 68 & 10630.000 & 25.171 & 24.915 & 82.647 & 82.625 \\
\hline 69 & 10772.000 & 25.061 & 24.752 & 82.620 & 82.792 \\
\hline 70 & 10914.000 & 24.835 & 24.596 & 83.114 & 82.952 \\
\hline 71 & 11125.000 & 24.707 & 24.375 & 83.350 & 83.179 \\
\hline 72 & 11267.000 & 24.650 & 24.232 & 83.417 & 83.324 \\
\hline 73 & 11408.000 & 24.304 & 24.097 & 83.398 & 83.463 \\
\hline 74 & 11600.000 & 23.861 & 23.920 & 83.453 & 83.644 \\
\hline 75 & 11742.000 & 23.994 & 23.795 & 83.619 & 83.771 \\
\hline 76 & 11884.000 & 23.869 & 23.675 & 83.913 & 83.894 \\
\hline 77 & 12026.000 & 23.872 & 23.559 & 84.418 & 84.012 \\
\hline 78 & 12215.000 & 23.282 & 23.412 & 84.592 & 84.162 \\
\hline 79 & 12357.000 & 23.251 & 23.306 & 84.519 & 84.270 \\
\hline 80 & 12499.000 & 22.984 & 23.205 & 85.053 & 84.374 \\
\hline 81 & 12683.000 & 22.779 & 23.079 & 85.371 & 84.502 \\
\hline 82 & 12825.000 & 22.377 & 22.986 & 85.350 & 84.597 \\
\hline 83 & 12968.000 & 23.047 & 22.895 & 85.752 & 84.689 \\
\hline 84 & 13148.000 & 22.435 & 22.787 & 85.637 & 84.800 \\
\hline 85 & 13290.000 & 22.402 & 22.705 & 85.618 & 84.883 \\
\hline 86 & 13433.000 & 21.844 & 22.625 & 85.830 & 84.964 \\
\hline 87 & 13575.000 & 22.182 & 22.549 & 86.116 & 85.041 \\
\hline 88 & 13761.000 & 21.769 & 22.454 & 86.187 & 85.138 \\
\hline 89 & 13904.000 & 21.900 & 22.384 & 86.250 & 85.209 \\
\hline 90 & 14047.000 & 21.883 & 22.317 & 86.537 & 85.277 \\
\hline
\end{tabular}


Figure S1. First 1D WET ${ }^{1} \mathrm{H}$ NMR spectrum of the reaction for Table 1 calculations

First 1D WET ${ }^{1} \mathrm{H}$ NMR spectrum with integral ratios of substrate and product

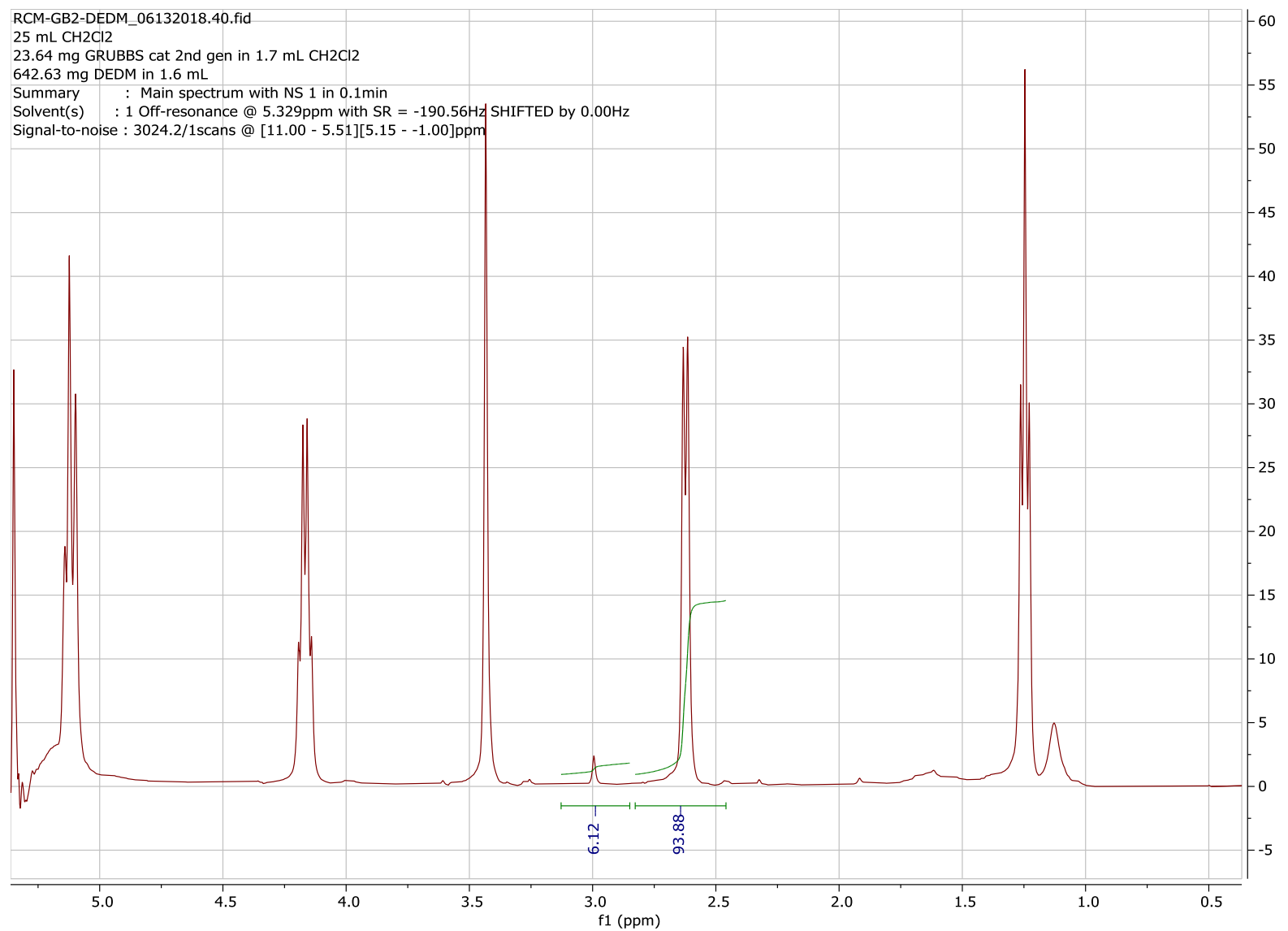


Table S2. Table of data for kinetic rate calculations for Table 1 based on integral ratios from the first spectrum

Table of data for kinetic rate calculations using Mnova software when concentration of the substrate and the product are adjusted based on their integral ratio from the first spectrum. The results of the rate constants are in Table 1 in the manuscript.

\begin{tabular}{|c|c|c|c|c|c|}
\hline $\begin{array}{l}\# \\
\text { (spectra) }\end{array}$ & $\begin{array}{l}\mathrm{X}(\mathrm{I}) \text { (time in } \\
\mathrm{sec})\end{array}$ & $\begin{array}{l}\mathrm{Y}(\mathrm{X}) \text { (concentration of } \\
\text { substrate in } \mathrm{mM} \text { ) }\end{array}$ & $\begin{array}{l}Y^{\prime}(X) \text { (fitted } \\
\text { data) }\end{array}$ & $\begin{array}{l}\mathrm{Y} 1(\mathrm{X}) \text { (concentration of } \\
\text { product in } \mathrm{mM} \text { ) }\end{array}$ & $\begin{array}{l}Y 1^{\prime}(X) \text { (fitted } \\
\text { data) }\end{array}$ \\
\hline Model & ARR_DATA(I) & Concentration $(2.751,2.489)$ & $\begin{array}{l}B+F^{*} \exp (- \\
\left.x^{*} G\right) \\
B=20.1071 \\
F=71.3459 \\
G= \\
0.000271534\end{array}$ & Concentration $(3.101,2.871)$ & $\begin{array}{l}B+F^{*} \exp (- \\
\left.x^{*} G\right) \\
B=80.7743 \\
F=-74.1679 \\
G= \\
0.000274149\end{array}$ \\
\hline 1 & 0.000 & 88.720 & 91.453 & 5.380 & 6.606 \\
\hline 2 & 146.000 & 90.503 & 88.680 & 8.766 & 9.516 \\
\hline 3 & 289.000 & 87.182 & 86.068 & 11.674 & 12.256 \\
\hline 4 & 496.000 & 83.653 & 82.463 & 15.947 & 16.036 \\
\hline 5 & 640.000 & 80.720 & 80.072 & 18.242 & 18.542 \\
\hline 6 & 782.000 & 78.520 & 77.804 & 20.845 & 20.918 \\
\hline 7 & 924.000 & 75.382 & 75.621 & 23.232 & 23.203 \\
\hline 8 & 1128.000 & 72.194 & 72.630 & 26.505 & 26.335 \\
\hline 9 & 1271.000 & 70.101 & 70.630 & 28.552 & 28.428 \\
\hline 10 & 1413.000 & 68.413 & 68.719 & 30.367 & 30.426 \\
\hline 11 & 1607.000 & 65.540 & 66.224 & 33.164 & 33.034 \\
\hline 12 & 1750.000 & 63.848 & 64.468 & 35.008 & 34.870 \\
\hline 13 & 1893.000 & 62.658 & 62.778 & 37.063 & 36.634 \\
\hline 14 & 2087.000 & 60.149 & 60.589 & 39.399 & 38.921 \\
\hline 15 & 2229.000 & 58.191 & 59.057 & 41.095 & 40.519 \\
\hline 16 & 2372.000 & 57.733 & 57.574 & 42.927 & 42.066 \\
\hline 17 & 2514.000 & 56.371 & 56.157 & 44.437 & 43.544 \\
\hline 18 & 2715.000 & 54.382 & 54.242 & 46.384 & 45.540 \\
\hline 19 & 2857.000 & 53.076 & 52.951 & 47.908 & 46.885 \\
\hline 20 & 2999.000 & 51.785 & 51.709 & 48.952 & 48.179 \\
\hline 21 & 3189.000 & 50.096 & 50.120 & 50.763 & 49.834 \\
\hline 22 & 3331.000 & 48.982 & 48.984 & 51.874 & 51.015 \\
\hline 23 & 3473.000 & 47.792 & 47.892 & 52.808 & 52.151 \\
\hline 24 & 3662.000 & 46.641 & 46.502 & 54.120 & 53.597 \\
\hline 25 & 3803.000 & 45.407 & 45.511 & 55.102 & 54.627 \\
\hline 26 & 3946.000 & 44.459 & 44.543 & 56.015 & 55.632 \\
\hline 27 & 4087.000 & 43.681 & 43.625 & 56.830 & 56.586 \\
\hline 28 & 4293.000 & 42.269 & 42.346 & 58.112 & 57.914 \\
\hline 29 & 4435.000 & 41.244 & 41.505 & 58.986 & 58.787 \\
\hline 30 & 4577.000 & 40.684 & 40.695 & 59.688 & 59.626 \\
\hline 31 & 4780.000 & 39.469 & 39.591 & 60.621 & 60.771 \\
\hline 32 & 4923.000 & 38.597 & 38.849 & 61.287 & 61.540 \\
\hline 33 & 5065.000 & 38.118 & 38.140 & 61.889 & 62.274 \\
\hline 34 & 5251.000 & 37.387 & 37.252 & 62.968 & 63.194 \\
\hline 35 & 5394.000 & 36.485 & 36.599 & 63.321 & 63.870 \\
\hline 36 & 5537.000 & 36.158 & 35.971 & 64.086 & 64.520 \\
\hline 37 & 5679.000 & 35.402 & 35.371 & 64.561 & 65.140 \\
\hline 38 & 5885.000 & 34.317 & 34.541 & 65.668 & 65.999 \\
\hline 39 & 6027.000 & 34.497 & 33.995 & 66.198 & 66.563 \\
\hline 40 & 6169.000 & 33.369 & 33.469 & 66.671 & 67.106 \\
\hline 41 & 6361.000 & 33.103 & 32.791 & 67.397 & 67.807 \\
\hline 42 & 6503.000 & 32.289 & 32.311 & 67.868 & 68.302 \\
\hline 43 & 6645.000 & 32.102 & 31.849 & 68.144 & 68.778 \\
\hline 44 & 6836.000 & 31.402 & 31.256 & 68.902 & 69.390 \\
\hline
\end{tabular}




\begin{tabular}{|c|c|c|c|c|c|}
\hline 45 & 69978.000 & 31.146 & 30.834 & 69.224 & 69.825 \\
\hline 46 & 7120.000 & 30.574 & 30.428 & 69.730 & 70.243 \\
\hline 47 & 7262.000 & 29.797 & 30.038 & 69.972 & 70.645 \\
\hline 48 & 7461.000 & 29.788 & 29.516 & 70.696 & 71.183 \\
\hline 49 & 7604.000 & 29.341 & 29.157 & 70.962 & 71.551 \\
\hline 50 & 7747.000 & 28.897 & 28.813 & 71.167 & 71.906 \\
\hline 51 & 7933.000 & 28.337 & 28.384 & 71.597 & 72.347 \\
\hline 52 & 8075.000 & 28.339 & 28.071 & 72.084 & 72.669 \\
\hline 53 & 8217.000 & 27.662 & 27.770 & 72.104 & 72.978 \\
\hline 54 & 8412.000 & 27.105 & 27.374 & 72.868 & 73.384 \\
\hline 55 & 8554.000 & 27.247 & 27.100 & 72.948 & 73.666 \\
\hline 56 & 8697.000 & 27.086 & 26.833 & 73.210 & 73.939 \\
\hline 57 & 8839.000 & 26.407 & 26.579 & 73.399 & 74.200 \\
\hline 58 & 9037.000 & 26.434 & 26.240 & 74.002 & 74.548 \\
\hline 59 & 9179.000 & 25.959 & 26.008 & 74.263 & 74.785 \\
\hline 60 & 9322.000 & 25.689 & 25.783 & 74.475 & 75.016 \\
\hline 61 & 9518.000 & 25.654 & 25.489 & 74.837 & 75.317 \\
\hline 62 & 9660.000 & 25.326 & 25.286 & 74.798 & 75.525 \\
\hline 63 & 9803.000 & 25.248 & 25.088 & 75.239 & 75.727 \\
\hline 64 & 10003.000 & 25.048 & 24.825 & 75.939 & 75.996 \\
\hline 65 & 10145.000 & 24.920 & 24.647 & 75.881 & 76.179 \\
\hline 66 & 10288.000 & 24.694 & 24.474 & 76.256 & 76.355 \\
\hline 67 & 10430.000 & 24.690 & 24.309 & 76.367 & 76.524 \\
\hline 68 & 10630.000 & 24.284 & 24.086 & 76.797 & 76.751 \\
\hline 69 & 10772.000 & 23.957 & 23.936 & 76.756 & 76.905 \\
\hline 70 & 10914.000 & 24.109 & 23.791 & 77.228 & 77.052 \\
\hline 71 & 11125.000 & 24.077 & 23.586 & 77.381 & 77.262 \\
\hline 72 & 11267.000 & 23.727 & 23.454 & 77.421 & 77.396 \\
\hline 73 & 11408.000 & 23.538 & 23.329 & 77.399 & 77.524 \\
\hline 74 & 11600.000 & 22.933 & 23.165 & 77.487 & 77.690 \\
\hline 75 & 11742.000 & 23.157 & 23.049 & 77.677 & 77.808 \\
\hline 76 & 11884.000 & 22.977 & 22.938 & 78.017 & 77.921 \\
\hline 77 & 12026.000 & 23.193 & 22.831 & 78.399 & 78.030 \\
\hline 78 & 12215.000 & 22.695 & 22.695 & 78.598 & 78.169 \\
\hline 79 & 12357.000 & 22.361 & 22.597 & 78.487 & 78.268 \\
\hline 80 & 12499.000 & 22.300 & 22.503 & 79.009 & 78.364 \\
\hline 81 & 12683.000 & 22.123 & 22.386 & 79.355 & 78.483 \\
\hline 82 & 12825.000 & 21.889 & 22.300 & 79.282 & 78.570 \\
\hline 83 & 12968.000 & 22.440 & 22.216 & 79.636 & 78.655 \\
\hline 84 & 13148.000 & 21.892 & 22.116 & 79.555 & 78.757 \\
\hline 85 & 13290.000 & 21.985 & 22.040 & 79.481 & 78.834 \\
\hline 86 & 13433.000 & 21.183 & 21.966 & 79.703 & 78.909 \\
\hline 87 & 13575.000 & 21.765 & 21.896 & 79.867 & 78.980 \\
\hline 88 & 13761.000 & 21.059 & 21.808 & 80.044 & 79.069 \\
\hline 89 & 13904.000 & 21.338 & 21.743 & 80.059 & 79.135 \\
\hline 90 & 14047.000 & 21.308 & 21.681 & 80.334 & 79.198 \\
\hline
\end{tabular}


Figure S2. Stack 1D WET ${ }^{1} \mathrm{H}$ NMR spectra and kinetic graph for Table 1 calculations based on integral ratios from the first spectrum

Stack 1D ${ }^{1} \mathrm{H}$ WET NMR spectra and kinetic graph using Mnova software when concentration of the substrate and the product are adjusted based on their integral ratio from the first spectrum. The results of the rate constants are in Table 1 in the manuscript.
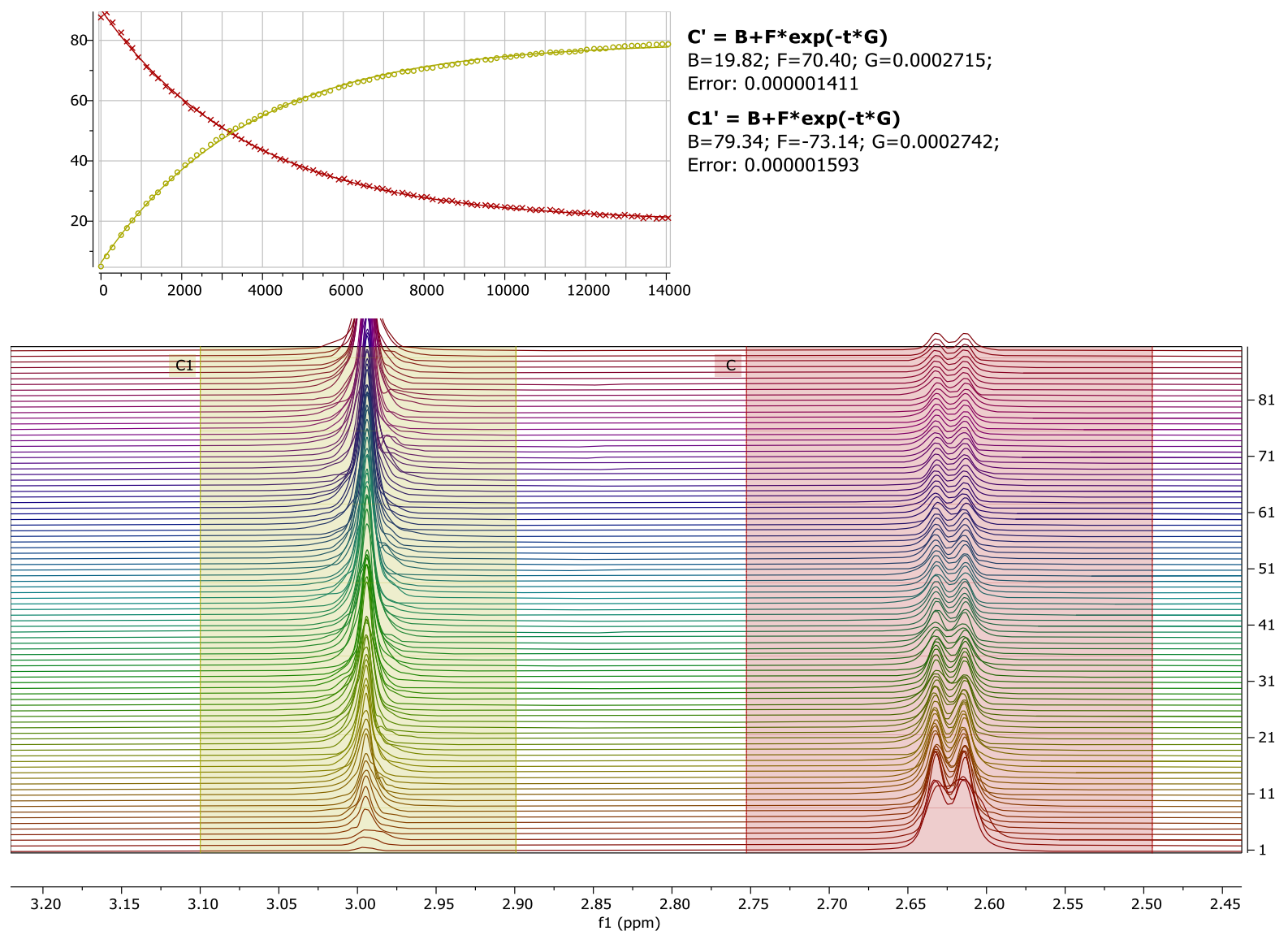
Figure S3. Last 1D WET ${ }^{1} \mathrm{H}$ NMR spectrum of the reaction for Table 1 calculations

Last 1D WET ${ }^{1} \mathrm{H}$ NMR spectrum with integral ratios of substrate and product

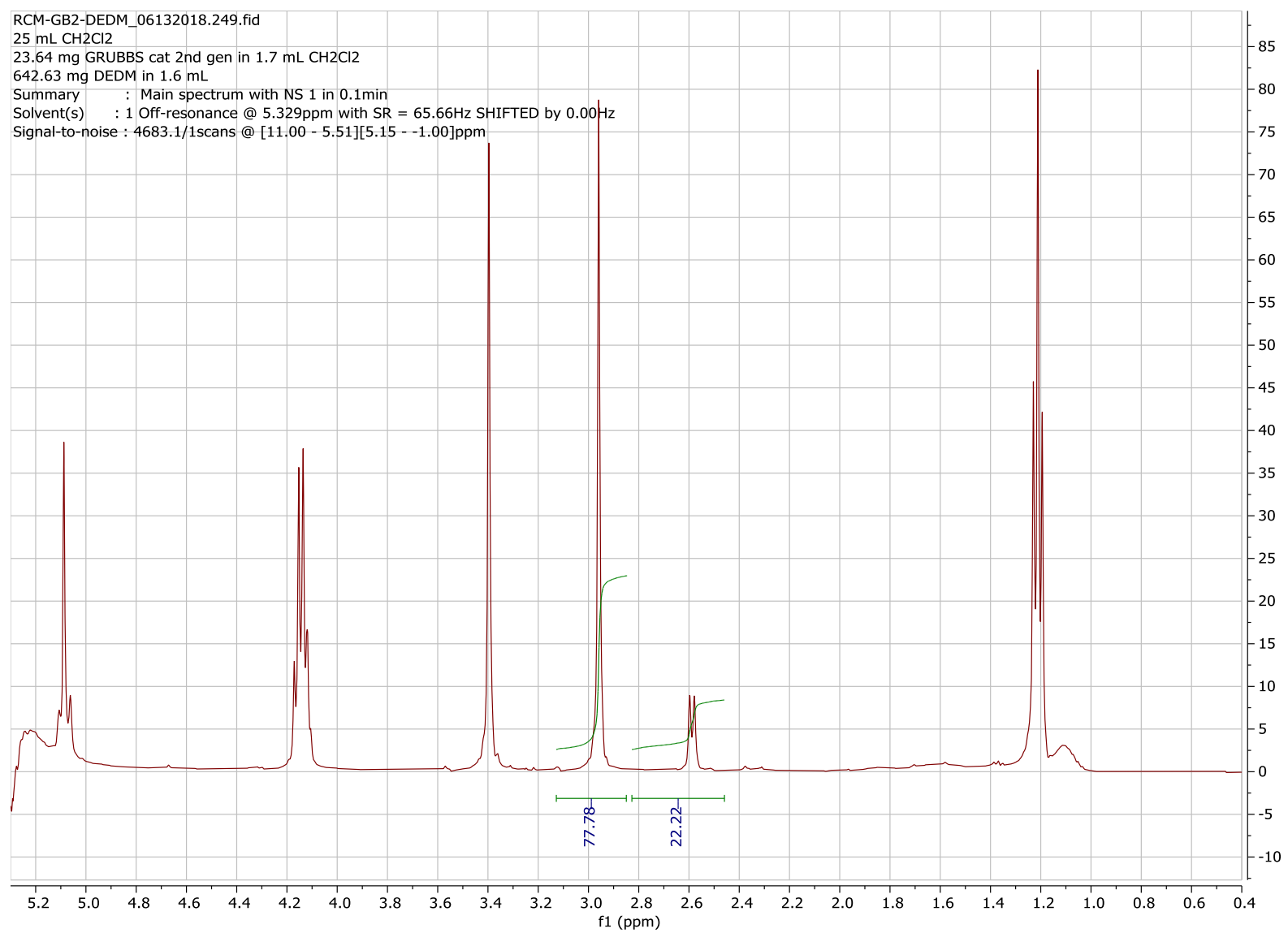


Table S3. Table of data for kinetic rate calculations for Table 1 based on integral ratios from the last spectrum

Table of data for kinetic rate calculations using Mnova software when concentration of the starting material and the product are adjusted based on their integral ratio from the last spectrum. The results of the rate constants are in Table 1 in the manuscript.

\begin{tabular}{|c|c|c|c|c|c|}
\hline $\begin{array}{l}\# \\
\text { (spectra) }\end{array}$ & $\begin{array}{l}X(\mathrm{I}) \text { (time in } \\
\mathrm{sec})\end{array}$ & $\begin{array}{l}\mathrm{Y}(\mathrm{X}) \text { (concentration of } \\
\text { substrate in } \mathrm{mM} \text { ) }\end{array}$ & $\begin{array}{l}\mathrm{Y}^{\prime}(\mathrm{X}) \text { (fitted } \\
\text { data) }\end{array}$ & $\begin{array}{l}\mathrm{Y} 1(\mathrm{X}) \text { (concentration of } \\
\text { product in } \mathrm{mM} \text { ) }\end{array}$ & $\begin{array}{l}\text { Y1'(X) (fitted } \\
\text { data) }\end{array}$ \\
\hline Model & ARR_DATA(I) & Concentration $(2.754,2.492)$ & $\begin{array}{l}B+F^{*} \exp (- \\
\left.x^{*} G\right) \\
B=19.8201 \\
F=70.396 \\
G= \\
0.000271468\end{array}$ & Concentration $(3.101,2.896)$ & $\begin{array}{l}B+F^{*} \exp (- \\
\left.x^{*} G\right) \\
B=79.3425 \\
F=-73.1431 \\
G= \\
0.000274223\end{array}$ \\
\hline 1 & 0.000 & 87.568 & 90.216 & 5.025 & 6.199 \\
\hline 2 & 146.000 & 89.236 & 87.481 & 8.288 & 9.070 \\
\hline 3 & 289.000 & 85.979 & 84.904 & 11.230 & 11.772 \\
\hline 4 & 496.000 & 82.539 & 81.348 & 15.423 & 15.501 \\
\hline 5 & 640.000 & 79.624 & 78.989 & 17.679 & 17.973 \\
\hline 6 & 782.000 & 77.423 & 76.752 & 20.235 & 20.317 \\
\hline 7 & 924.000 & 74.415 & 74.599 & 22.622 & 22.571 \\
\hline 8 & 1128.000 & 71.197 & 71.648 & 25.787 & 25.660 \\
\hline 9 & 1271.000 & 69.144 & 69.674 & 27.880 & 27.724 \\
\hline 10 & 1413.000 & 67.462 & 67.789 & 29.605 & 29.695 \\
\hline 11 & 1607.000 & 64.688 & 65.328 & 32.455 & 32.268 \\
\hline 12 & 1750.000 & 62.995 & 63.595 & 34.211 & 34.078 \\
\hline 13 & 1893.000 & 61.855 & 61.928 & 36.267 & 35.818 \\
\hline 14 & 2087.000 & 59.355 & 59.768 & 38.589 & 38.073 \\
\hline 15 & 2229.000 & 57.384 & 58.258 & 40.204 & 39.650 \\
\hline 16 & 2372.000 & 56.936 & 56.794 & 41.962 & 41.176 \\
\hline 17 & 2514.000 & 55.584 & 55.396 & 43.438 & 42.634 \\
\hline 18 & 2715.000 & 53.599 & 53.507 & 45.354 & 44.602 \\
\hline 19 & 2857.000 & 52.355 & 52.233 & 46.914 & 45.929 \\
\hline 20 & 2999.000 & 51.126 & 51.007 & 48.052 & 47.205 \\
\hline 21 & 3189.000 & 49.455 & 49.439 & 49.856 & 48.837 \\
\hline 22 & 3331.000 & 48.302 & 48.319 & 50.806 & 50.002 \\
\hline 23 & 3473.000 & 47.168 & 47.242 & 51.790 & 51.122 \\
\hline 24 & 3662.000 & 45.968 & 45.870 & 52.997 & 52.548 \\
\hline 25 & 3803.000 & 44.753 & 44.892 & 53.935 & 53.564 \\
\hline 26 & 3946.000 & 43.872 & 43.937 & 54.993 & 54.555 \\
\hline 27 & 4087.000 & 43.114 & 43.032 & 55.789 & 55.495 \\
\hline 28 & 4293.000 & 41.676 & 41.769 & 56.966 & 56.805 \\
\hline 29 & 4435.000 & 40.664 & 40.939 & 57.931 & 57.666 \\
\hline 30 & 4577.000 & 40.112 & 40.141 & 58.498 & 58.494 \\
\hline 31 & 4780.000 & 38.929 & 39.051 & 59.481 & 59.623 \\
\hline 32 & 4923.000 & 38.073 & 38.319 & 60.125 & 60.381 \\
\hline 33 & 5065.000 & 37.582 & 37.619 & 60.688 & 61.105 \\
\hline 34 & 5251.000 & 36.892 & 36.743 & 61.794 & 62.012 \\
\hline 35 & 5394.000 & 35.995 & 36.098 & 62.137 & 62.678 \\
\hline 36 & 5537.000 & 35.645 & 35.479 & 62.704 & 63.319 \\
\hline 37 & 5679.000 & 34.930 & 34.887 & 63.349 & 63.931 \\
\hline 38 & 5885.000 & 33.819 & 34.067 & 64.359 & 64.778 \\
\hline 39 & 6027.000 & 34.010 & 33.528 & 64.837 & 65.334 \\
\hline 40 & 6169.000 & 32.916 & 33.010 & 65.537 & 65.869 \\
\hline 41 & 6361.000 & 32.669 & 32.340 & 66.156 & 66.560 \\
\hline 42 & 6503.000 & 31.842 & 31.867 & 66.563 & 67.048 \\
\hline 43 & 6645.000 & 31.673 & 31.411 & 66.897 & 67.518 \\
\hline 44 & 6836.000 & 30.979 & 30.825 & 67.700 & 68.121 \\
\hline 45 & 6978.000 & 30.738 & 30.409 & 68.043 & 68.550 \\
\hline 46 & 7120.000 & 30.154 & 30.009 & 68.452 & 68.962 \\
\hline
\end{tabular}




\begin{tabular}{|c|c|c|c|c|c|}
\hline 47 & 7262.000 & 29.377 & 29.624 & 68.672 & 69.358 \\
\hline 48 & 7461.000 & 29.388 & 29.108 & 69.534 & 69.888 \\
\hline 49 & 7604.000 & 28.938 & 28.754 & 69.753 & 70.252 \\
\hline 50 & 7747.000 & 28.488 & 28.414 & 69.889 & 70.602 \\
\hline 51 & 7933.000 & 27.956 & 27.991 & 70.450 & 71.036 \\
\hline 52 & 8075.000 & 27.951 & 27.682 & 70.759 & 71.353 \\
\hline 53 & 8217.000 & 27.284 & 27.385 & 70.827 & 71.659 \\
\hline 54 & 8412.000 & 26.706 & 26.995 & 71.419 & 72.059 \\
\hline 55 & 8554.000 & 26.868 & 26.723 & 71.637 & 72.337 \\
\hline 56 & 8697.000 & 26.710 & 26.461 & 71.909 & 72.606 \\
\hline 57 & 8839.000 & 26.059 & 26.209 & 72.258 & 72.864 \\
\hline 58 & 9037.000 & 26.043 & 25.875 & 72.519 & 73.206 \\
\hline 59 & 9179.000 & 25.593 & 25.646 & 73.007 & 73.440 \\
\hline 60 & 9322.000 & 25.337 & 25.424 & 73.176 & 73.667 \\
\hline 61 & 9518.000 & 25.296 & 25.134 & 73.593 & 73.964 \\
\hline 62 & 9660.000 & 24.986 & 24.933 & 73.584 & 74.170 \\
\hline 63 & 9803.000 & 24.914 & 24.738 & 74.086 & 74.369 \\
\hline 64 & 10003.000 & 24.700 & 24.478 & 74.494 & 74.634 \\
\hline 65 & 10145.000 & 24.573 & 24.302 & 74.482 & 74.814 \\
\hline 66 & 10288.000 & 24.335 & 24.132 & 74.816 & 74.988 \\
\hline 67 & 10430.000 & 24.345 & 23.968 & 75.033 & 75.154 \\
\hline 68 & 10630.000 & 23.935 & 23.749 & 75.292 & 75.378 \\
\hline 69 & 10772.000 & 23.620 & 23.601 & 75.462 & 75.529 \\
\hline 70 & 10914.000 & 23.756 & 23.458 & 75.787 & 75.675 \\
\hline 71 & 11125.000 & 23.755 & 23.255 & 76.080 & 75.881 \\
\hline 72 & 11267.000 & 23.379 & 23.125 & 75.912 & 76.013 \\
\hline 73 & 11408.000 & 23.221 & 23.001 & 76.214 & 76.140 \\
\hline 74 & 11600.000 & 22.621 & 22.840 & 76.216 & 76.304 \\
\hline 75 & 11742.000 & 22.835 & 22.725 & 76.288 & 76.420 \\
\hline 76 & 11884.000 & 22.655 & 22.616 & 76.612 & 76.531 \\
\hline 77 & 12026.000 & 22.868 & 22.510 & 76.903 & 76.639 \\
\hline 78 & 12215.000 & 22.371 & 22.375 & 77.211 & 76.775 \\
\hline 79 & 12357.000 & 22.051 & 22.279 & 77.231 & 76.873 \\
\hline 80 & 12499.000 & 21.965 & 22.186 & 77.367 & 76.968 \\
\hline 81 & 12683.000 & 21.795 & 22.070 & 77.734 & 77.085 \\
\hline 82 & 12825.000 & 21.577 & 21.985 & 77.791 & 77.171 \\
\hline 83 & 12968.000 & 22.122 & 21.903 & 78.099 & 77.254 \\
\hline 84 & 13148.000 & 21.583 & 21.804 & 78.162 & 77.355 \\
\hline 85 & 13290.000 & 21.691 & 21.729 & 78.160 & 77.431 \\
\hline 86 & 13433.000 & 20.871 & 21.656 & 78.267 & 77.504 \\
\hline 87 & 13575.000 & 21.478 & 21.586 & 78.606 & 77.575 \\
\hline 88 & 13761.000 & 20.761 & 21.500 & 78.619 & 77.662 \\
\hline 89 & 13904.000 & 21.041 & 21.436 & 78.677 & 77.727 \\
\hline 90 & 14047.000 & 21.000 & 21.374 & 78.694 & 77.789 \\
\hline
\end{tabular}


Figure S4. Stack 1D WET ${ }^{1} H$ NMR spectra and kinetic graph for Table 1 calculations based on integral ratios from the last spectrum

Spectra and kinetic graph using Mnova software when concentration of the substrate and the product are adjusted based on their integral ratio from the last spectrum. The results of the rate constants are in Table 1 in the manuscript.
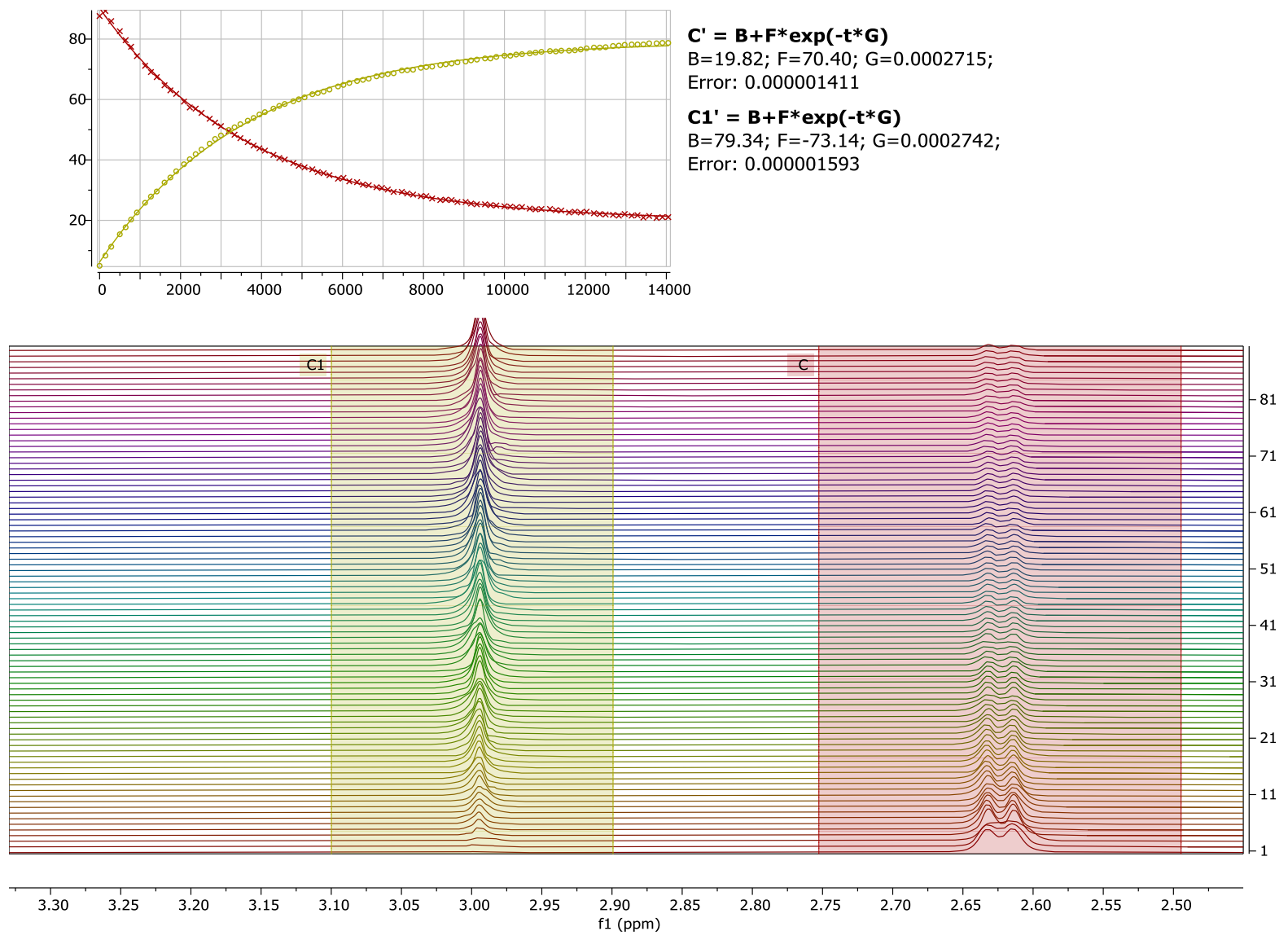
Figure S5. Results from Bruker Dynamic Center software calculations for Table 1

Next pages depict the results of the calculations done with Dynamic Center Bruker software (rate constants are in Table 1 in the manuscript)

\begin{tabular}{|l|l|}
\hline Fitted function alternatives: & $\begin{array}{l}f(t)=10^{*} \exp (-k t) \\
f(t)=10^{*} \exp (-k t)+C \\
f(t)=10^{*}[1-\exp (-k t)] \\
f(t)=10 * \text { * } 1-\exp (-k t)]+C\end{array}$ \\
\hline Random error estimation of data: & RMS per spectrum (or trace/plane) \\
\hline Systematic error estimation of data: & worst case per peak scenario \\
\hline Fit parameter Error estimation method: & from fit using calculated y uncertainties \\
\hline Confidence level: & $95 \%$ \\
\hline Used peaks: & peaks from peaklist.xml at spectrum \\
\hline Used integrals: & area integral \\
\hline Used time: & all values (including replicates) used \\
\hline
\end{tabular}

$f(t)=10 * \exp (-k t)+C$

\begin{tabular}{|c|c|c|c|c|c|c|c|}
\hline name & F2 [ppm] & offset & Error & lo & Error & $\mathrm{k}[1 / \mathrm{s}]$ & Error \\
\hline 2 & 2.622 & $8.47 \mathrm{e}+05$ & 198.6 & $3.42 \mathrm{e}+06$ & 314.1 & 0.000270 & $6.385 \mathrm{e}-08$ \\
\hline
\end{tabular}

$f(t)=10 *[1-\exp (-k t)]+C$

\begin{tabular}{|c|c|c|c|c|c|c|c|}
\hline name & F2 [ppm] & offset & Error & lo & Error & $k[1 / \mathrm{s}]$ & Error \\
\hline 1 & 2.992 & $1.83 \mathrm{e}+05$ & 333.0 & $3.55 \mathrm{e}+06$ & 298.4 & 0.000276 & $5.872 \mathrm{e}-08$ \\
\hline
\end{tabular}

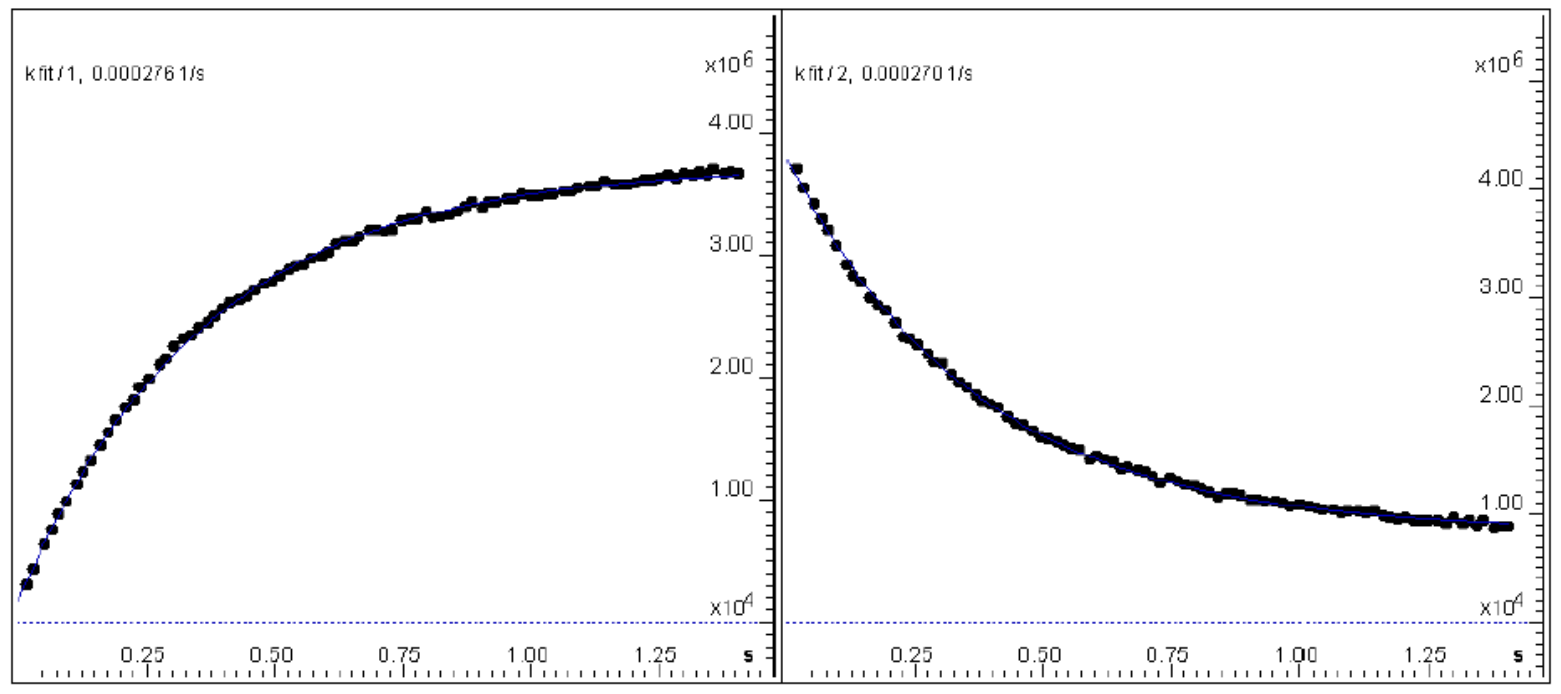


Current fit display

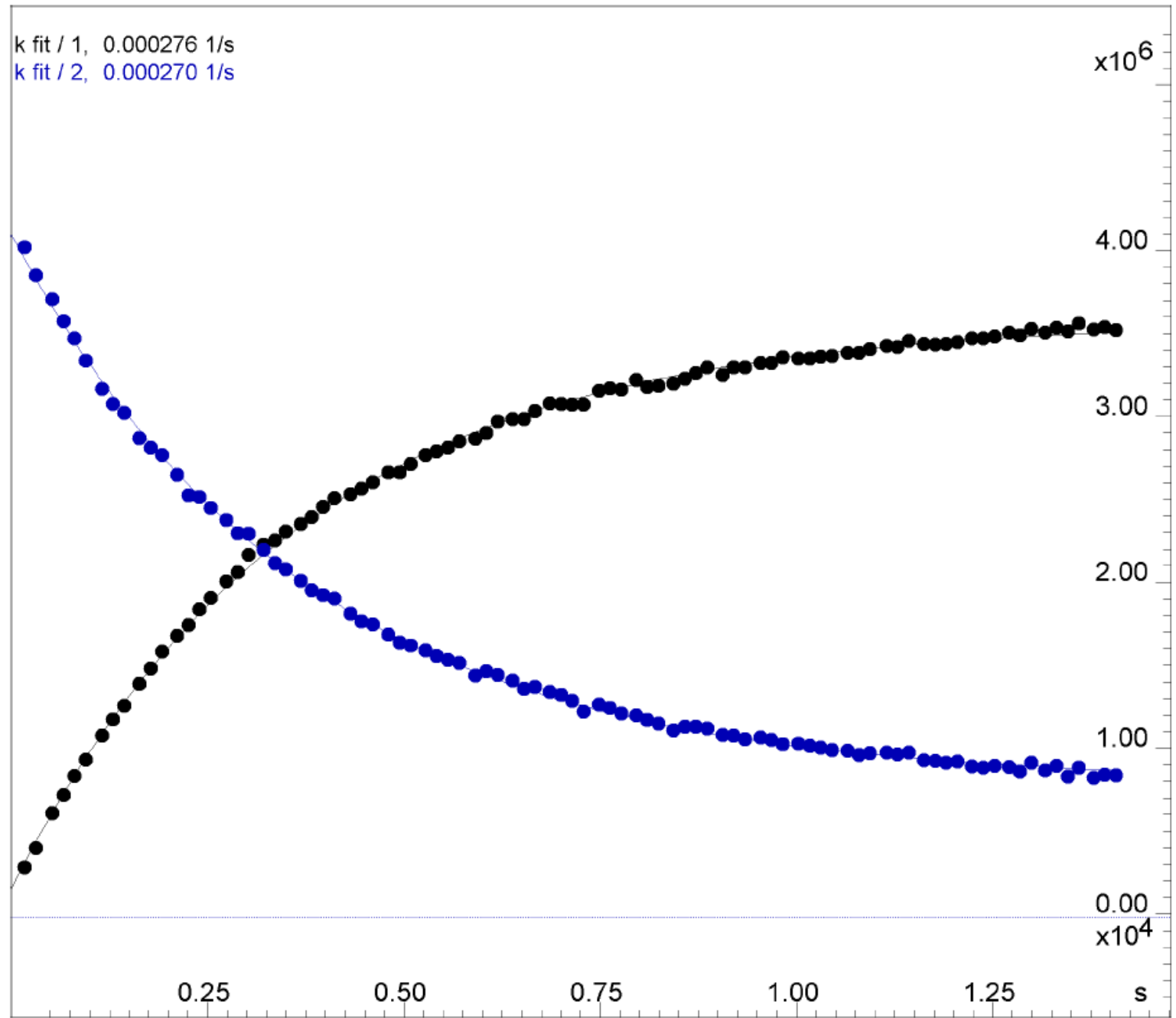


1D ${ }^{1} \mathrm{H}$ NMR spectrum with the signal selected for the calculations.

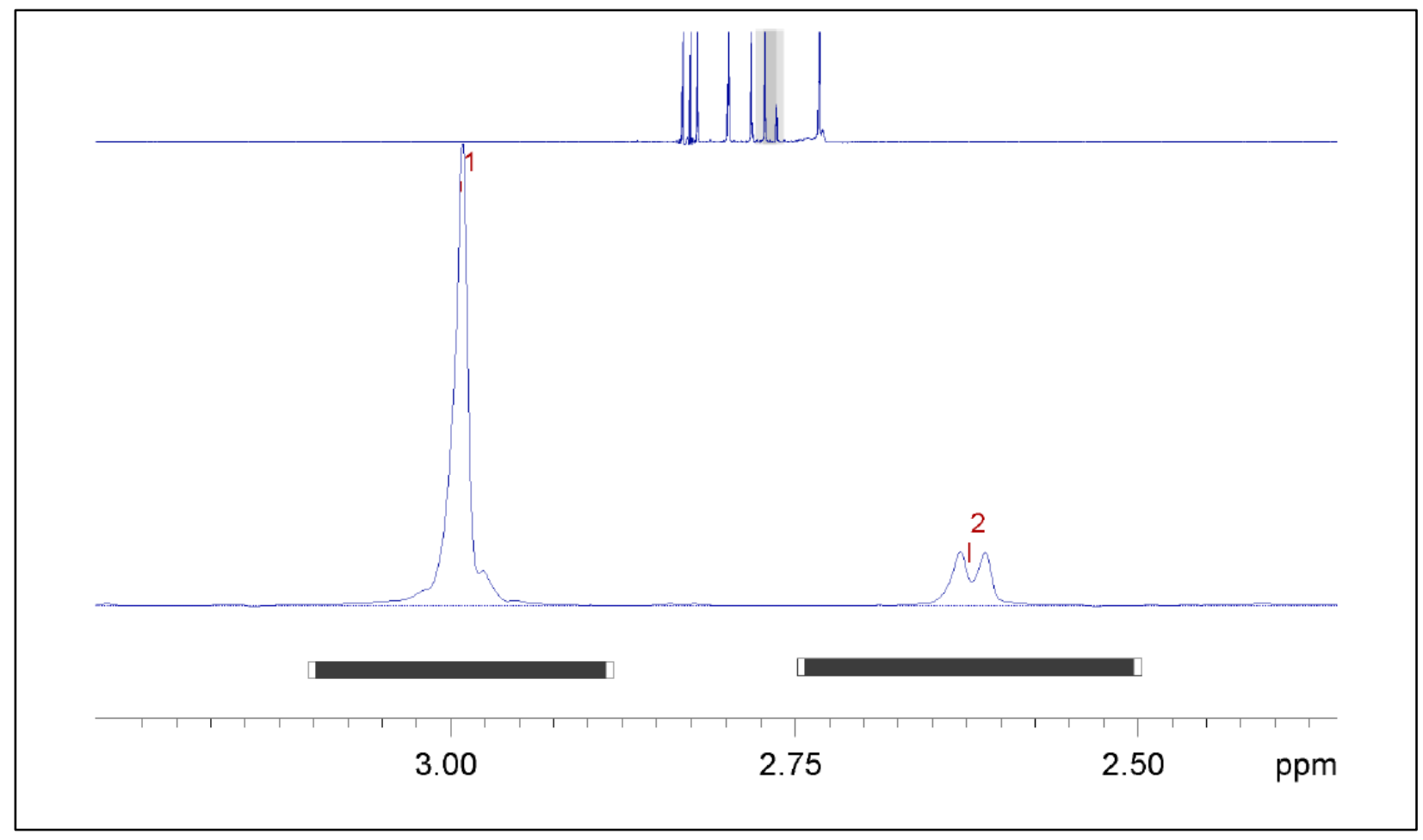

